\title{
AS DIRETRIZES PARA DESENVOLVIMENTO DA PRESTAÇÃO DE SERVIÇO DE MANUTENÇÃO DE EDIFICAÇÕES EM INSTITUIÇÃO PỦBLICA DE ENSINO
}

\author{
Francisco Tadeu de Almeida Magalhães (UFF - Universidade Federal Fluminense) \\ tadeu.magalhaes@ hotmail.com.br
Julio Vieira Neto (UFF - Universidade Federal Fluminense)
julion@id.uff.br

Resumo: Objetiva-se nesta pesquisa, identificar os fatores envolvidos com a gestão da manutenção predial em instituições públicas com foco nas instituições de ensino, a partir de um controle efetivo do processo da manutenção desde sua solicitação pelo serviço, até sua conclusão satisfatória, pelos procedimentos adotados, a iniciar pela solicitação do serviço, passos adotados para preparo da execução, disponibilidades para a execução e a própria execução. Pretende-se verificar diretrizes que possam ser aplicadas à forma como se presta esse serviço, no sentido melhorar o acompanhamento, tanto quanto melhorar as relações internas entre o prestador do serviço da manutenção e solicitante. Verificando a literatura disponível sobre gestão da manutenção predial, procurou-se dar foco na influência do acompanhamento do processo na qualidade da prestação do serviço e na relação entre os setores envolvidos, com ênfase nos solicitantes e nos prestadores do serviço. Assim percebe-se a necessidade de adoção de diretrizes que orientem esse processo, levando às melhorias citadas.

Palavras-chaves: Manutenção predial; Gestão de manutenção predial; Gestão da manutenção. 


\section{Introdução}

Verifica-se no processo de prestaçõa de serviço na manutenção de instalações prediais de instituiçoes públicas, o foco do prestador do serviço em solucionar o problema "reclamado" sem envolvimento com os desdobramentos ligados a esse trabalho. Dentre esses desdobramentos, é passivo encontrar-se situações onde o setor prestador do serviço, não tenha pessoal disponível para o atendimento, ou não disponha de material consumível para a aplicação, outros fatores que possam habilitar o atendimento.

A falta de disponibilizar essa informação para o solicitante do serviço (principalmente), pode permitir interpretações errôneas quanto a gestão do setor prestador do serviço, tanto quanto a possibilidade do prestador do serviço ser objeto de incômodos por ansiedade ou necessidade do solicitante para a respectiva correção.

Para tanto pretende-se avaliar as implicações resultantes dessa relação solicitante versus prestador, assim como a qualidade da prestação com a possibilidade da melhoria da relação entre esses dois protagonistas.

Ao final desse trabalho, pretende-se fazer uma verificação dos fatores que derivam da de um bom trabalho na prestação de serviços, envolvendo desde os aspectos tecnicoadministrativos e a relação que é estabelecida entre o prestador do serviço e o solicitante desde uma comunicação efetiva entre ambos.

Nos permitindo uma breve retrospectiva; Milan e Ribeiro (2003) já apontavam para a busca do marketing de relacionamento conjugado a retenção de clientes, como agentes de sucesso das empresas

Ainda Milan e Ribeiro (2003), citam empresas que apresentaram ganhos entre $25 \%$ e $85 \%$ e reduzindo a perda de clientes em $5 \%$. Citam ainda a valorização da atividade de pósvendas ligadas ao negócio, como prática de marketing que retém o cliente ou usuário pelo sentimento de satisfação, onde o desafio é fazer este usuário ter certeza de sua importância na medida e cofia no relacionamento continuado pós contratação.

Já Gouveia; Pinto; Oliveira (2015), citam a terceirização no setor público das atividades de apoio, dentre as quais se destaca a manutenção predial na Reforma Administrativa de 1995; e um pouco mais adiante, Ramos (2019) ressalta a importância de trabalho denominado pós-vendas, estabelecendo que o relacionamento se potencializa, após a venda (pós-venda) e ajuda a determinar a escolha do cliente na próxima compra; definindo assim a importância da atividade de prestação do serviço ao usuário. 
O desempenho e a satisfação no trabalho como um componente da motivação, gerando a eficiência e o aumento da produtividade de um colaborador, além da permanência na empresa (turnover) é comentado por Assunção (2018). Assim, a má iluminação, um equipamento deficiente para uso em trabalho, um mobiliário avariado, dentre outros agentes que possam comprometer a infraestrutura, podem influenciar negativamente nesses fatores.

Pode-se estabelecer a manutenção predial, como "o conjunto de atividades e recursos que garanta o melhor desempenho da edificação para atender às necessidades dos usuários, com confiabilidade e disponibilidade, ao menor custo possível” Gomide et al. (2006). Uma vez que no contexto de custo, Aziz; Nawawi; Ariff - 2016, cita que de acordo com o Instituto Nacional de Tecnologia Científica do E.U.A., dois terços do custo estimado em uma edificação são perdidos devido a ineficiência durante a fase de operação e manutenção.

Já para Caldas (2014), os ativos intangíveis, promovem perspectivas de benefícios futuros, até mesmo financeiros. Desta forma, a prestação do serviço, no caso em específico deste trabalho, a manutenção prestada em instalações prediais com uma boa relação entre solicitante e prestador, tende a criar valores que se enquadram na classe dos intangíveis.

Destaca Carlino (2012), que o setor público não vivencia a competitividade e nele não existe a distinção de funcionários quanto a produtividade, tal como isso é acentuado no setor privado. Nessa linha, em órgãos públicos um plano ou programa que poderíamos entender diretamente como manutenção predial, prevê manter a qualidade dos serviços de manutenção prestados para a preservação do patrimônio público derivando na diminuição de gastos desnecessários.

Em complemento, Dost et al (2016), define a formação de capital social de uma organização como "conhecimento intrínseco à organização e que é utilizado na interação entre os indivíduos e suas relações”. Em complemento, Olanrewaju, A. Abdul Lateef (2012), cita que estudos confirmaram o desempenho dos edifícios educacionais e a qualidade da educação desenvolvem correlações positivas

Para isso, é importante entender o desenrolar desse processo desde o seu início incluindo as etapas mais significativas de cada passo desenvolvido. Buscando simplificar de forma objetiva o "Fluxograma de Atendimento ás Solicitações de Manutenção" baseado em similar proposto por Morais e Lordsen Jr. (2016), observamos a seguinte sequência operacional, tal como apresentado no fluxograma da Fig. 1 
Figura 1 - Fluxo de operação em uma solicitação de serviços

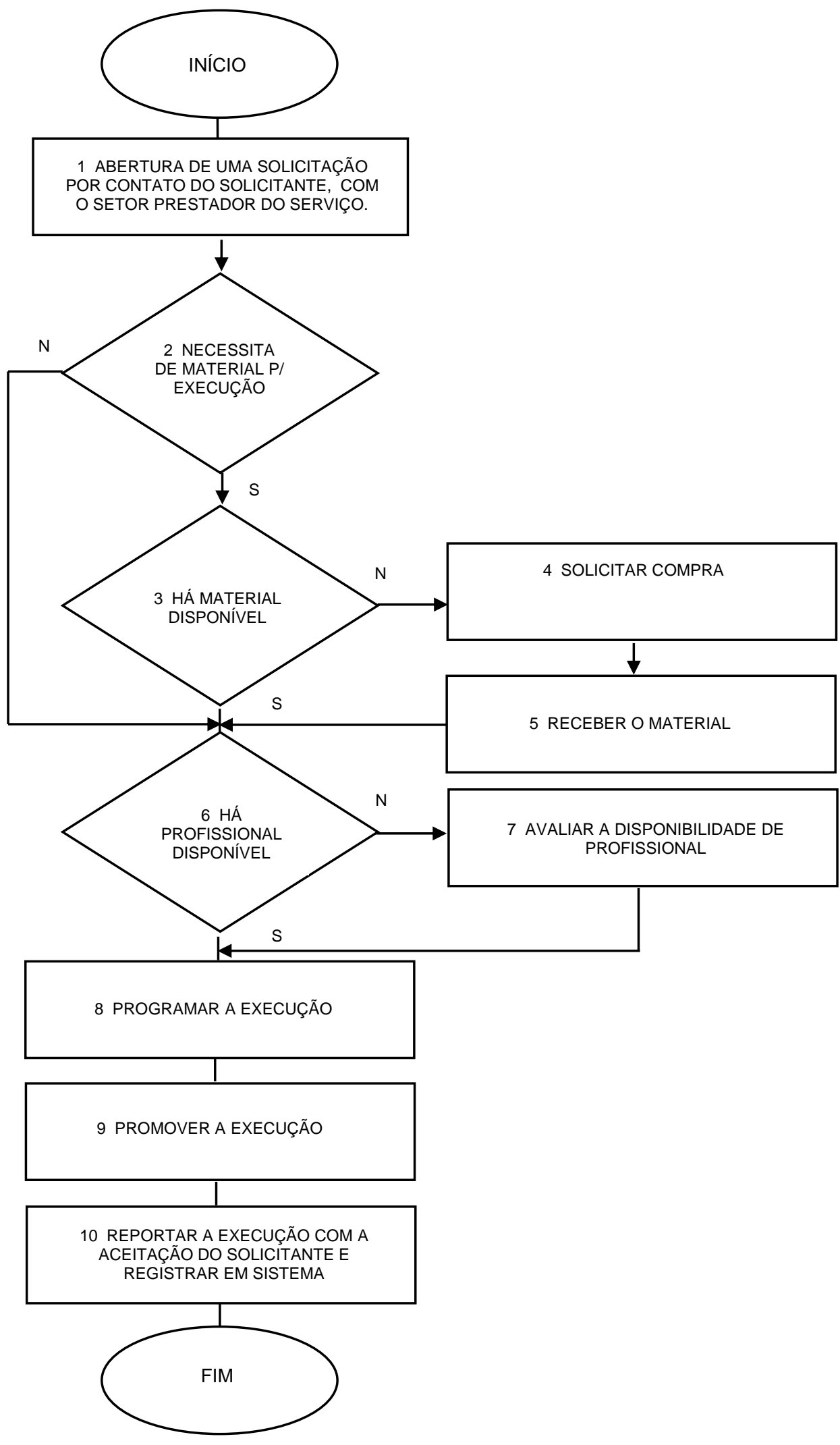

Fonte: Elaboração própria 
Verifica-se no fluxograma da fig. 1 algumas variáveis que podem influenciar diretamente no atendimento, impondo maior demora ou mais agilidade, levando o prestador de serviço a ser impedido de prestar os serviço com a rapidez que pretende e ao solicitante ser submetido a um retardo no atendimento desconhecendo a razão deste, como aborda Conte (2016), observados os blocos 4, 5 e 7 do fluxograma da fig. 1, se apresentam como agentes influenciadores externos ao setor de prestação do serviço.

O fluxo de etapas básicas apresentados na Fig. 1, poderá ser objeto de revisão para verificação de possibilidade de incrementos em sua sequência de ocorrências em abordagens futuras quando aplicado um planejamento de logística interno ao setor prestador do serviço, mas ainda assim deverão seguir exercendo sua influência no tempo final desta prestação..

\section{Metodologia}

Procurou-se dar seguimento, buscando essas publicações e promovendo a verificação de suas aderências ao tema proposto para desenvolvimento, fazendo uso daquelas que realmente pudessem contribuir com o trabalho. Nessa mesma linha de procedimento, verificou-se as publicações editoriais, algumas não tão recentes porém não menos relevantes, que pudessem contribuir para o desenvolvimento do trabalho

Promovendo-se a busca usando-se os mesmos critérios, aplicados a palavras chave; Com os resultados apresentados, ao serem focados os resultados mais antigos, observa-se números que podem conduzir a publicações com considerável nível de obsolescência. Por esta possibilidade, procurou-se restringir a busca, inicialmente, publicações que não fossem anteriores a 2015; o que já permitiu números um mais reduzidos.

Observou-se que na filtragem realizada, o número retornos obtidos ainda representavam números elevados uma vez que retornavam publicações ligadas a áreas de pouco ou nenhum interesse à proposta do trabalho. Selecionando então as áreas de interesse e envolvimento com o trabalho, promoveu-se a busca restringindo-se as áreas de engenharia, gestão de negócios e contas, e ciências sociais.

Verifica-se também, que um tema mais abrangente como a gestão da manutenção (Maintenance Management), já alcança números excessivamente elevados, mesmo quando foram usadas restrições específicas por área de publicação. Assim mesmo, não seria possível desconsiderar essa busca, uma vez que sua aderência ao tema do trabalho mostra-se de grande importância e relevância. 


\section{Análise dos Resultados}

Ao citar a emissão de relatórios periódicos com lista dos serviços pendentes como proposto por Morais e Lordsleen Jr. (2018), já prevê um passo inicial para o acompanhamento da prestação de serviços de manutenção, que permita ao solicitante um seguimento à sua solicitação de forma a melhorar a relação entre solicitante e prestador do serviço e com isso, agregar a instituição valores intangíveis gerados por melhores relações entre seus departamentos, setores ou pessoas.

Enfatizado por Assunção (2018) que, ainda não sendo muitas as publicações, verifica-se como a satisfação e o comprometimento do trabalho ligados com a eficiência, aumento do desempenho, produtividade. Pode-se identificar que esses fatores se originam em relacionamentos de qualidade entre pessoas, setores, departamentos, divisões e empresas. Tratamos então de um processo que se inicia a partir da prestação do serviço da manutenção baseado num canal de comunicação entre os prestadores do serviço o os solicitantes.

Alguns itens recorrentes preocupados com a melhoria operacional ligadas a um sistema de manutenção em instalações, onde Atkin e Bildsten (2017) citam dentre esses itens; o estado dos ambientes internos e o local de trabalho versus a produtividade do trabalhador, incluindo também a disponibilidade em bom funcionamento dos recursos tecnológicos.

A gestão do processo da manutenção exige acompanhamentos permanentes, tanto das estruturas em manutenção quanto dos procedimentos para sua execução. Frangopol; Saydam e Sunyong (2012), discorrem sobre a necessidade de se manter indicadores de desempenho de um sistema, capazes de fornecerem informações sobre a interação entre os fatores que compõem a manutenção e o desempenho geral dessa manutenção

Procedimentos adotados mediante critérios de estratégia de ações de manutenção, induzem ao desenvolvimento de um serviço prestado com qualidade em suas características técnicas e satisfação do solicitante, como aborda Carreira (2018), ressaltando não só a prática consagrada e tradicional do P.D.C.A. $($ Plan $=$ Planejar, Do $=$ Executar, Check $=$ Avaliar, Act = Agir) como mais que isso, conduz ao uso do S.L.A. (Service Level Agreement $=$ Tratado de Nível de Serviço); com atividades definidas em um nivelamento de significância.

Tal como observado por Zawawia; Kamaruzzamab; Ithnina; Zulkarnaina (2011); os gestores da prestação de serviços estiveram preocupados em prestar os serviços de forma reativa em vez de prestar os serviços de forma proativa, desconsiderando a satisfação dos solicitantes (clientes) do serviço, causando mau impacto para os serviços prestados. 
Ainda de acordo com Carreira (2018), essas práticas conduzirão a um plano de especificidades que irão compor o SLA de forma a orientar para conclusão satisfatória tecnicamente e atendendo a expectativa de seu solicitante. Como exemplo, apresenta o quadro da fig. 2.

Figura 2 - Especificidades que podem conter num SLA

\begin{tabular}{|c|c|}
\hline \multicolumn{2}{|c|}{ ESPECIFICIDADE QUE DEVEM CONTER NUM SLA COMPLETO } \\
\hline SERVIÇO & GESTÃO \\
\hline $\begin{array}{l}\text { Definir com clareza quais os serviços a prestar, } \\
\text { sem que exista espaço para dúvidas; }\end{array}$ & Definição de padrões e métodos de medição de indicadores; \\
\hline Condições de disponibilidade do serviço; & Definição de processos de resolução de litigios; \\
\hline $\begin{array}{l}\text { Tempo de revisão para cada alteraçãa dos } \\
\text { niveis de serviço; }\end{array}$ & $\begin{array}{l}\text { Definição de cláusulas de indenização com a intenção de } \\
\text { proteger o cliente; }\end{array}$ \\
\hline Definição das responsabilidades. & Definição de mecanismos de atualização do contrato. \\
\hline
\end{tabular}

Fonte: CORREIA 2018 - Adaptado de Maurício (2011)

A qualidade da prestação do serviço na manutenção está ligada diretamente a sua excelente prática na gestão dos procedimentos adotados. Isso leva não só a satisfação do usuário solicitante, como também ao aumento do ciclo de vida dos sistemas, tal como aborda Zawawia; Kamaruzzamab; Ithnina; Zulkarnaina (2011), minimizando falhas inesperadas ou deteriorações precoces.

A gestão da manutenção de uma instalação, está diretamente comprometida com o desempenho e o relacionamento de seus ocupantes; de acordo com a abordagem de Soh; Chua; Ali; Au-Young and Alias (2018), a manutenção de edifícios deve ter sua gestão combinando ações técnicas e administrativas capazes de garantir que as partes de um edifício e suas instalações, cumpram um padrão necessário e aceitável em seu desempenho.

Os programas de manutenção devem buscar a qualidade em seus resultados, como propõe Frangopol; Saydam e Sunyong (2012), destacando a necessidade de se prever o 
desempenho dos sistemas em função de seus ciclos de vida, quando aplicáveis, e com isso estabelecer programas de manutenção eficazes e ideais obtendo desses sistemas seus melhores desempenhos.

Todo esse processo se destaca a partir de um planejamento da prestação de serviço que possa contemplar etapas bem definidas e seus respectivos acompanhamentos. Um bom planejamento, evita erros na etapa de execução, como aborda Valle (2019) (apud Vargas 2005), buscando minimizar erros nas execuções oriundos de falhas na etapa de planejamento.

\section{Conclusões}

Por todos os pontos que nos foi permitido focar o tema, verifica-se a existência sempre permanente, da necessidade de instrumentos que permitam um canal de comunicação entre solicitante do serviço e seu prestador. Vemos em diversas abordagens que os prestadores de serviço de manutenção, principalmente em instituições públicas de ensino, em sua maioria, deixam de manter contato adequado com seus solicitantes, pelas razões mais diversas.

Os fatores que dificultam a comunicação, são alegados por cada caso em particular, mas o mais comum, é que a maioria desses setores só se encontram disponíveis para manutenção, em peíodo de férias escolares, feriados longos, ou em situações muito particulares de cada instituição.

Analisando pelo "ângulo oposto", no período em que esses ambientes estão em uso, a presença do profissional de manutenção é dificultada, quando não impossibilitada, por estarem os ambientes em uso com docentes e discentes; salvo excessões para casos de manutenção emergencial tais como, de um vazamento hidráulico, sistema elétrico inoperante, impedem a utilização do ambiente e exigem atuação mais imediata do setor de manutenção.

Assim, é importante uma diretriz capaz de mantter um canal de comunicação mais ativo entre solicitante e prestador do serviço, ao longo de suas etapas (ver fig. 1), íncluindo possíveis variáveis externas capazes de alterar aquele fluxo, mantendo o solicitante sempre informado do desenvolvimento para a solução do problema, assim como ao prestador do serviço estar respaldado em alguma possível demora ou atraso na conclusão, levando o solicitante entender as possíveis razões recorrentes no processo e evitando avaliações distorcidas ou erradas a respeito do empenho do prestador do serviço. 


\section{Referências}

ASSUNÇÃO, Alynson Bruno Martins. Proposta de diretrizes para aumento da satisfação no trabalho e comprometimento organizacional: Contribuição de práticas de responsabilidade social e empresarial. Dissertação de Mestrado Sistema de Gestão, LATEC - Universidade Federal Fluminense. Rio de Janeiro. 2018

ATKIN, Brian; BILDSTEN, Louise. A future for facility management Department of Building and Environmental Technology.. Division of Construction Management, Lund University, Lund, Sweden, Process, Management. 2017 ISSN: 1471-4175

AZIZ, Nor Diana; NAWAWI, Abdul Hadi; ARIFF, Nor Rima Muhamad. Building information modelling (BIM) in facilities management: opportunities to be considered by facility managers. Procedia-Social and Behavioral Sciences - Elsevier, v. 234, p. 353-362, 2016.

CALDAS, Sergio Leal. Intangíveis - O Lado oculto da gestão empresarial. 1. ed. - Rio de Janeiro: Digitaliza. 2014. 184 p. ISBN 978-85-66959-02-4

CARLINO, A. E. Melhorias dos Processos de Manutenção em Prédios Públicos. Dissertação de Mestrado, Universidade Federal de São Carlos, São Paulo, 2012

CARREIRA, Maria Lidia Brito da Silva. Ferramentas do gerenciamento de facilidades aplicadas à gestão da manutenção predial. Gestão e gerenciamento, [S.1.], v. 9, n. 9, p. 22-31, set. 2019. ISSN 2447-1291. Disponível em:

https://nppg.org.br/revistas/gestaoegerenciamento/article/view/329. Acesso em: 15/11/2019

CONTE, Francesco; Proposta de um modelo de gestão da manutenção com qualidade total para escolas de nível superior - estudo de caso do CEFET/RJ. Dissertação apresentada ao Curso de Mestrado em Sistemas de Gestão. LATEC - Universidade Federal Fluminense. 2016

DOST, Mir; BADIR, Yuorsre F.; ALI Zeeshan; TARIQ Aeel. The impact of intellectual capital on innovation generation and adoption. Journal of Intellectual Capital. 2016. ISSN: 1469-1930

FRANGOPOL, Dan M.; SAYDAM, Duygu; KIM, Sunyong. Maintenance, management, lifecycle design and performance of structures and infrastructures: a brief review. Journal Structure and infrastructure engineering, v. 8, n. 1, p. 1-25. 2012. Disponível em: https://www.tandfonline.com/doi/abs/10.1080/15732479.2011.628962. Acessado em: $15 / 09 / 2019$

GOMIDE, Tito Lívio Ferreira. Profissionalismo na Construção Civil em tempos de Certificação do Desempenho Edilício. Instituto de Engenharia - SP 2015, disponível em https://www.institutodeengenharia.org.br/site/2015/01/06/profissionalismo-na-construcaocivil-em-tempos-de-certificacao-do-desempenho-edilicio/. Acessado em: 20/07/2019

GOUVÊA, Maria Aparecida; PINTO, Ricardo Lopes; OLIVEIRA, Braulio. Avaliação da qualidade de serviços terceirizados de intervenção em mobiliário e no layout em uma organização pública. Revista de Administração da Universidade Federal de Santa Maria, v.8, n. 1, p. 103-124. 2015. Disponível em:

https://www.redalyc.org/pdf/2734/273441378007.pdf. Acessado em: 22/072019

MILAN, Gabriel Sperandio; RIBEIRO, José Luis Duarte. A influência do departamento de pós-vendas na retenção de clientes: um estudo qualitativo em uma empresa de medicina de 
grupo. Gest. Prod., São Carlos , v. 10, n. 2, p. 197-216, Aug. 2003 . Disponível em: $<$ http://www.scielo.br/scielo.php?script=sci_arttext\&pid=S0104530X2003000200006\&lng=en\&nrm=iso>. Acessado em: 20/07/2019

MORAIS, Gabriela Alves Tenório de; JÚNIOR, Alberto Casado Lordsleem. Building maintenance management activities in a public institution. Engineering, Construction and Architectural Management, v. 26, n. 1, p. 85-103. 2019.

OLAREWAJU, A. Abdul Lateef. Quantitative analysis of defects in university buildings: user perspective Built Environment Project and Asset Management. 2012. Disponível em: www.emeraldinsight.com/2044-124X.htm. Acessado em: 28/09/2019

RAMOS, Lucas Adriano Dias. Vitrine de Insatisfação: Um olhar para o pós-vendas das relações comerciais de eletrônicos em plataformas digitais. Revista Estudos e Pesquisas em Administração - ISSN 2594-7559. Universidade Federal de Mato Grosso. Campus Universitário de Rondonópolis, Mato Grosso, Brasil. Curso de Administração 2019

SOH, Khai Ying; CHUA, Shirley Jin Lin; ALI, Azlan Shah; AU-YOUNG, Cheong Peng; LIAS, Anuar. Relationship between building maintenance sourcing strategy selection factors and performance. Journal Facilities Management. 2019

VALLE, Lourenço Tostes. Avaliação de sistema de gerenciamento de projetos em instituição federal de ensino superior. Dissertação de Mestrado em Sistema de Gestão, LATEC - Universidade Federal Fluminense. 2019

ZAWAWI, E.M.A.; KAMARUZZAMANB, S.N.; ITHNIN Z.; ZULKARNAIN, S.H.. A Conceptual framework for describing CSF of building maintenance management. ELSEVIER - Procedia Engineering. Volume 20, 2011, Pages 110-117. 2011

ZGHID, Afef Benyoussef Zghidi. ZAIEM, Imed. Service orientation as a strategic marketing tool: the moderating effect of business sector, Competitiveness Review 27, pp. 40-61, 2017 